\title{
ACCESSORY FISSURES OF LIVER AND THEIR CLINICAL SIGNIFICANCE
}

\author{
Namrata Reddy'1, P. S. Mittal'2, S. S. Joshi³, S. D. Joshi
}

${ }^{1}$ Assistant Professor, Department of Anatomy, SAMC and PGI, Indore.

${ }^{2}$ Associate Professor, Department of Anatomy, KDMC and RC, Mathura.

3 Director and Professor, Department of Anatomy, SAMC and PGI, Indore.

${ }^{4}$ Director and Professor, Department of Anatomy, SAMC and PGI, Indore.

\section{ABSTRACT}

\section{BACKGROUND}

With the advent of USG, CT and MRI it is easy to visualise and localise the pathologies of liver, which is of great help in surgical interventions like resection and transplantation procedures.

Variations of fissures on the surfaces of liver in the Central Indian population have been observed in the present study, some of which are not in consonance with that described by other workers. In an era of segmental resections, transplantation and laparoscopic surgeries the findings of the present study can be of great significance.

\section{METHODS AND MATERIALS}

The work has been carried out on 52 cadaveric livers and a detailed observation of the surfaces of liver were done to find the presence of various fissures and sulci. These were measured, recorded and photographed.

\section{RESULTS}

On visceral surface of liver fissures were present: (a) To the right of porta hepatis in 53.8\%; (b) To the right of fossa for gallbladder in 25\% cases; (c) On the visceral surface of the left lobe in $7.6 \%$; (d) In 4 cases fissures were found to the right of the groove for inferior vena cava, (e) In $55.77 \%$ of livers the fissures were found on the quadrate lobe and (f) In $44.23 \%$ they were present on the caudate lobe. In $86.5 \%$, the fissure for Ligamentum Teres (LT) was cutting across the inferior border to extend to a variable distance on the anterior surface of the liver. Diaphragmatic furrows were found on anterosuperior surface of right lobe varying from 1 to 3 in number.

\section{CONCLUSIONS}

These fissures may be confused with cyst or haematoma and may be the site of implantation of secondaries. In an era of segmental resections, transplantation and laparoscopic surgeries the findings of the present study are of great significance.

\section{KEYWORDS}

Liver, Fissures, Ligamentum Teres, Diaphragmatic Furrows.

HOW TO CITE THIS ARTICLE: Reddy N, Mittal PS, Joshi SS, et al. Accessory fissures of liver and their clinical significance. J. Evolution Med. Dent. Sci. 2016;5(82):6125-6128, DOI: 10.14260/jemds/2016/1383

\section{BACKGROUND}

The liver is a glandular organ. It is the second largest single organ in the human body (after the skin) weighing approximately $1.5 \mathrm{~kg}$ in an average adult, comprising $1 / 50^{\text {th }}$ of total weight of the body. ${ }^{1}$ In man, the liver is essential for survival since there is currently no artificial organ or equipment that has the capacity to compensate for the absence of liver functions. In humans it consists of two major lobes that are subdivided into smaller segments. Morphological variations in the human liver have been classified as congenital or acquired. The complexity of liver function and its importance in body homeostasis has encouraged many anatomists to study the morphological features of the organ in considerable details. ${ }^{2}$ Accessory hepatic fissures are potential

Financial or Other, Competing Interest: None.

Submission 06-09-2016, Peer Review 30-09-2016,

Acceptance 06-10-2016, Published 12-10-2016.

Corresponding Author:

Dr. S. D. Joshi,

\#204, Sapna Apartment,

39 Kailash Park Colony,

Indore-452001.

E-mail: sdjoshi_2003@hotmail.com

DOI: $10.14260 /$ jemds/2016/1383

\section{(c) $($ ) $(-)$}

sources of diagnostic errors, both in sonography and CT. It is important to keep in mind the anomalies of liver during the preoperative diagnosis, because it will be helpful for the surgeons in planning biliary surgery or a portosystemic anastomosis. ${ }^{3}$

The peritoneum invaginates into the liver parenchyma normally or as an anatomic variation or pathologically leading to the formation of fissures and furrows. There are two normal fissures: fissure for the ligamentum teres and fissure for ligamentum venosum. Besides these, additional fissures may be found on the surface of liver. Fissures caused by normal anatomic variations include furrows that are created by diaphragmatic indentation and also accessory fissures that are found on the visceral surface. Pathologic fissures occur secondary to traumatic or iatrogenic causes or as a result of liver cirrhosis. When peritoneally disseminated tumour cells are implanted into these spaces, these may mimic intrahepatic focal lesions. ${ }^{4}$ When ascites or haemoperitoneum is loculated in the fissures or recesses, it may be mistaken for a liver cyst, intrahepatic haematoma or liver abscess. Since the clinical consequences for these entities are very different, exact localisation of the lesions may be crucial in the diagnosis and management of these lesions. ${ }^{5} \mathrm{~A}$ thorough understanding of the anatomy of liver surfaces and knowledge of location of 
these fissures and sulci may prevent a misdiagnosis of these focal lesions.

\section{MATERIALS AND METHODS}

Fifty two human livers available in the Dept. of Anatomy were used in the present study. The livers were apparently normal without any gross pathology. The age of the cadavers ranged from 50-70 years. The livers procured had the attachment of falciform ligament, coronary ligament and triangular ligament on its surface. The fissures present on the visceral surface of the right and left lobes were observed and their lengths were measured with digital Vernier callipers, scale and thread. The direction of fissures was noted (oblique, horizontal or vertical) in some livers. Extension of fissure for Ligamentum teres on the anterior surface of liver when present was measured. Furrows on the diaphragmatic surface of liver were observed; their different sites were noted, measured and tabulated. All the variations were recorded and photographed.

\section{RESULTS}

1. The average weight of liver was found to be $1.06 \mathrm{~kg}$ (range $0.46 \mathrm{~kg}-1.99 \mathrm{~kg}$ ).

2. The extension of fissure for ligamentum teres on the anterior - surface (Fig-1) was present in 45 livers, i.e. $86.5 \%$ and absent in 7 livers, i.e. - $13.5 \%$. The average length of extension of the fissure on ant. surface was 1.89 cm \{Range: $0.70-6.00 \mathrm{~cm}$.

3. a) The incidence of diaphragmatic furrows (Fig. 2) was $26.9 \%$ ( 14 livers). Of these $64.3 \%$ were single furrows; $28.6 \%$ showed two furrows and three furrows were found in $7.14 \%$ livers. b) In $57.1 \%$ these furrows were found on the anterosuperior surface of right lobe; in $28.6 \%$ they were present on the superior surface and in $7.14 \%$ of livers these were found on the right lateral surface. Remaining $7.14 \%$ showed it on the anterior surface of right-lobe. c) Mean length of the furrows was $4.58 \mathrm{~cm}$ (Range $0.5-5 \mathrm{~cm}$ ). d) The mean distance of the furrows from right layer of Falciform-ligament was 7.35 cm (Range 1.0 - $14.5 \mathrm{~cm}$ ).

4. Accessory Fissures

A.i) In 25 livers (53.8\%), the accessory fissures were present on the visceral surface of right lobe (Fig-3A) to the right of Porta-Hepatis (PH). Out of these in 32.6\% these fissures began just at the right end of $\mathrm{PH}$, whereas in $9.6 \%$ they were placed at a distance of $1-2 \mathrm{~cm}$ from right end of $\mathrm{PH}$. In $11.5 \%$, the fissures were curved or oblique. In one liver, two horizontal parallel fissures were found at the level of $\mathrm{PH}$. The average length of fissures to the right of porta hepatis was $2.82 \mathrm{~cm}$. (Range $0.5-5.0 \mathrm{~cm}$ ). ii) In 13 livers (25\%), the fissures were present to the right of fossa for Gallbladder (GB) (Fig. 3A). Out of these, in $56.2 \%$ the fissures were horizontal. In $37.5 \%$ they were curved and in only one liver it was vertical, lying parallel to GB. The mean length of these fissures was $3.25 \mathrm{~cm}$ (Range 1.1 - 8.0 $\mathrm{cm})$.
B. The fissures were observed on the visceral surface of left lobe (Fig. 3B). The incidence of these fissures was approximately $8 \%$. They were present at the level of porta hepatis in $4 \%$ of livers and in $2 \%$ they were related to the left margin of fissure for ligamentum teres. In the remaining 2\%, they were related to the left margin of fissure for ligamentum venosum. The mean length of fissure was $1.86 \mathrm{~cm}$ (Range $1-2.5 \mathrm{~cm}$ ) and the average distance of fissures from inferior margin was $4.1 \mathrm{~cm}$ (Range 2 - $6 \mathrm{~cm}$ ).

5. In $55.77 \%$ of liver fissures were present on the quadrate lobe (Fig. 4A), which were either obliquely directed or curved. The mean length of the fissures was $2.43 \mathrm{~cm}$ (Range $0.5-5.6 \mathrm{~cm}$ ) and mean distance from inferior border was $2.06 \mathrm{~cm}$ (Range $0.5-5.6 \mathrm{~cm}$ ).

6. Vertical or curved fissures were also present on the caudate lobe in $44.23 \%$ livers (Fig. 4B, 4C).

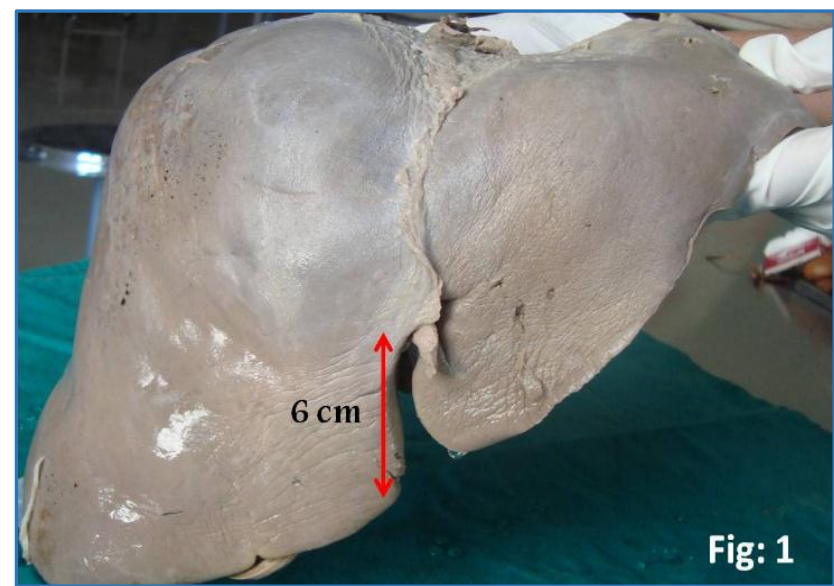

Fig. 1: Showing the Fissure for Ligamentum Teres Cutting through the Inferior Border of Liver and Extending $6 \mathrm{~cm}$ on the Anterior Surface

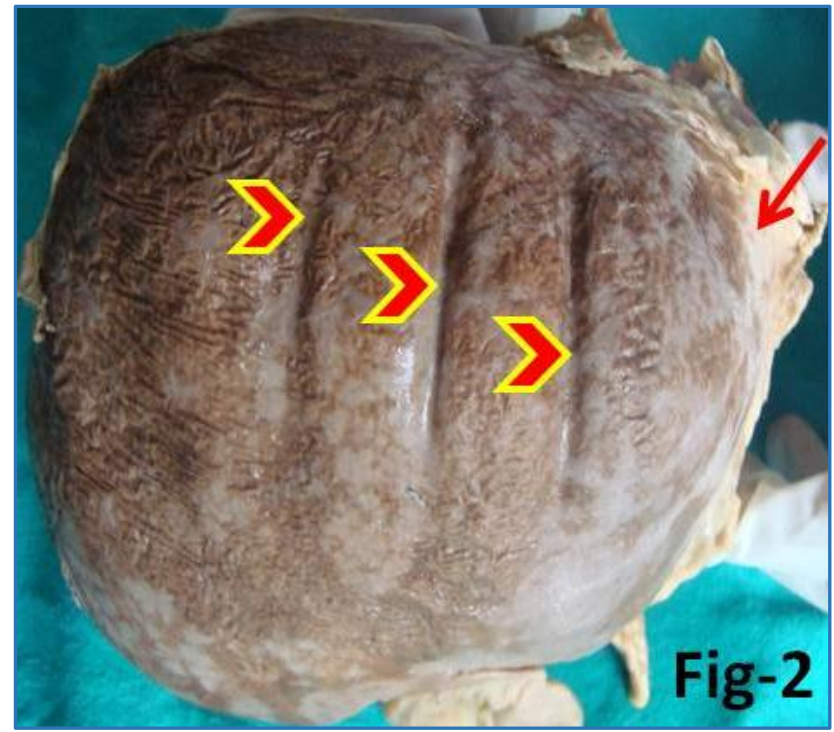

Fig. 2: Showing Three Long Vertical Diaphragmatic Furrows (Red Arrow Heads) on the Anterosuperior Surface of Right Lobe of Liver; also seen Falciform Ligament (Red Arrow). Furrows Lie at Variable Distance from the attachment of Falciform Ligament 


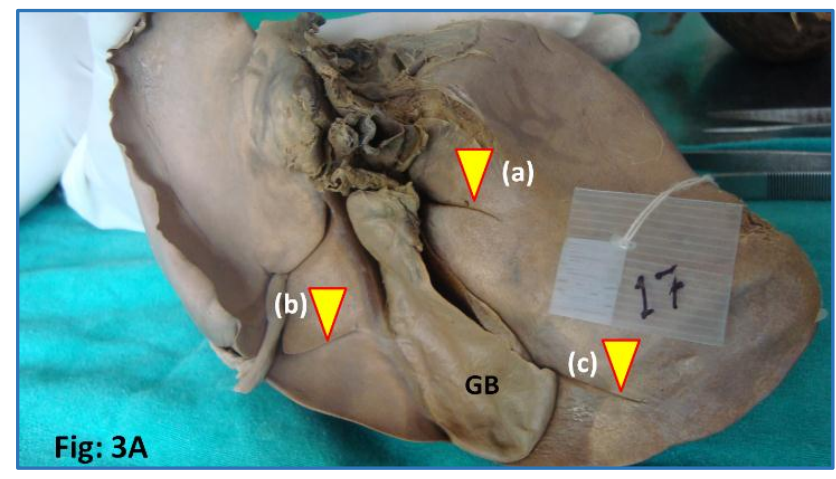

Fig. 3A: Visceral Surface of Right Lobe of Liver showing: Fissure to the Right of Porta Hepatis (a), Oblique Fissure Traversing the Quadrate Lobe of Liver (b), and Fissure on the Right Lobe to the Right of Fossa for Gallbladder (GB) - (c)

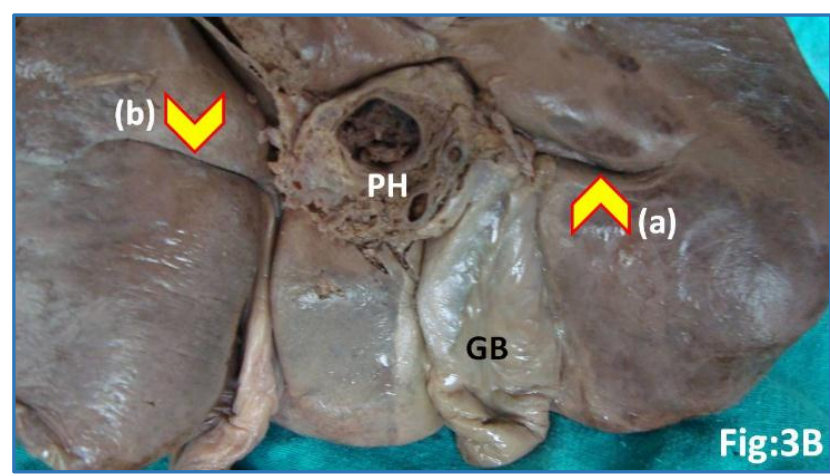

Fig. 3B: Shows Porta Hepatis (PH). (a) Fissure on the Right Lobe at the Level of Porta Hepatis. (b) Fissure on the Left Lobe to the Left of Porta Hepatis

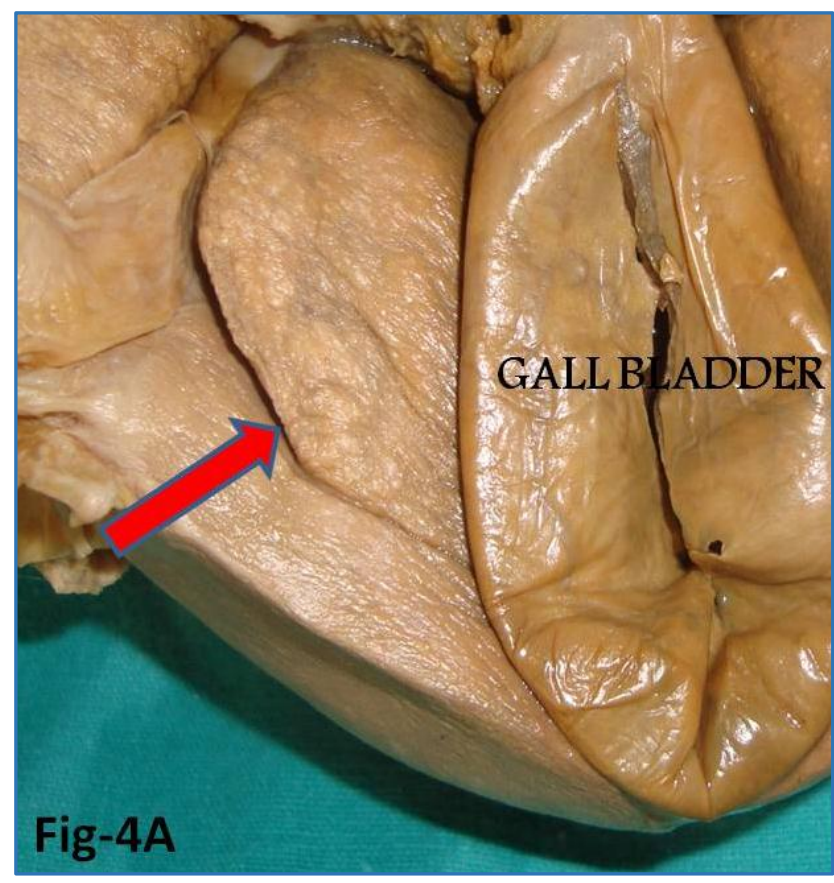

Fig. 4A: Showing a Curved Fissure on the Quadrate Lobe of Liver (Red Arrow)

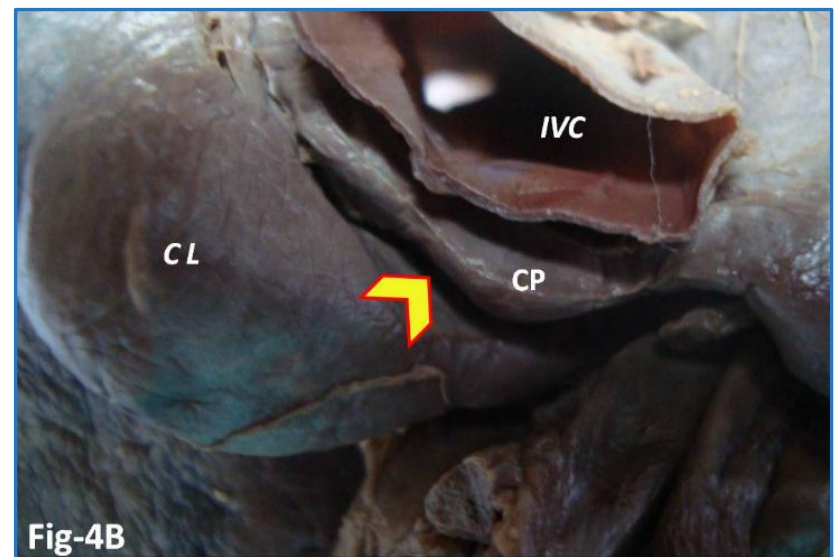

Fig. 4B: Showing a Deep Curved Fissure (Yellow Arrow Head). That is Separating Caudate Lobe (CL) from Caudate Process (CP). IVC = Inferior Vena Cava

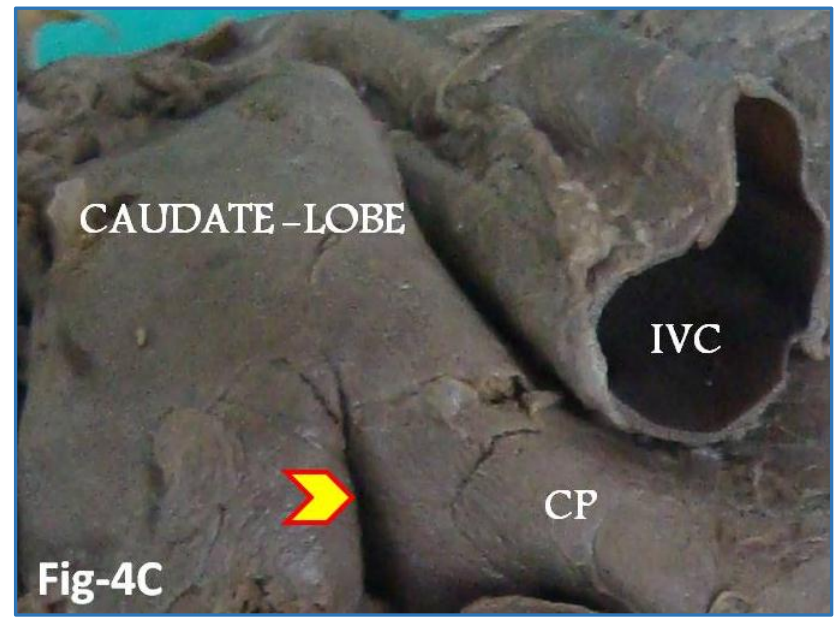

Fig. 4C: Showing a Deep Vertical Fissure (Yellow Arrow Head) Intervening between Caudate Lobe (CL) and Caudate Process (CP)

\section{DISCUSSION}

In our study, we have observed the presence of accessory fissures and furrows on 52 livers. Sahni et al in 1997 reported the average weight of liver in males and females as 1.5 and 1.3 kg respectively6; but in the present study, where we have not segregated livers into male and female, it was $1.06 \mathrm{~kg}$. This variation in the weight may be accounted for by the smaller built of the people of this region.

In place of a small notch at the inferior border of liver for the Ligamentum Teres (LT), majority of them showed a deep fissure cutting across the inferior border and extending to a variable length on the anterior surface of liver. Joshi et al (2009) ${ }^{5}$ showed the extension of this fissure for LT on the anterior surface in $80 \%$ cases, which is quite similar to our finding of $86.5 \%$ in the present study, whereas Nayak et al $(2013)^{7}$ reported its extension in only $1.81 \%$. The lack of description of such an extension of the fissure for LT on the anterior surface of liver warrants its incorporation in the text books of human anatomy.

Machhi et al $(2003)^{8}$ in a detailed description on the diaphragmatic sulci and portal fissures have stated that the presence of accessory sulci on the surface of liver had been known for a long time, but their possible clinical relevance as surface markings had not been suggested earlier. These sulci 
which are mainly located on the anterior and superior surface of right lobe are thought to be produced either by pressure of the ribs or the diaphragmatic muscle.

The incidence of diaphragmatic furrows as reported by Auh et al (1984) ${ }^{9}$ was $63 \%$. Machhi et al $(2003)^{8}$ reported its presence in $40 \%$ cases, whereas Joshi et al (2009) 5 found it in only $6 \%$ livers. Patil et al (2014) ${ }^{10}$ have mentioned its presence in only $2 \%$ cases and Saritha et al (2015) ${ }^{11}$ have reported it to be $4 \%$. In the present study, it was found in $27 \%$ livers. Muktyaz et al (2013) ${ }^{12}$ have reported a higher incidence in the autopsy material and also during radiological investigations. The apparent disparity in the findings of various workers may be attributable to various factors that may lead to its formation, viz. pressure of the ribs or the diaphragm on the weaker territories of the liver.

The mean length of these furrows was $4.6 \mathrm{~cm}$ in the present study which is less than $6-8 \mathrm{~cm}$ as observed by Shailaja et al (2011).13 The average distance of these furrows from right layer of falciform ligament was $7.35 \mathrm{~cm}$ in the present study, but no comparable data is available for this in the literature reviewed. Machhi et al $(2003)^{8}$ have put forward the explanation that the uneven growth of the liver parenchyma caused by the variable resistance by the different bundles of the diaphragmatic muscle may lead to the development of furrows and these may represent the weak zones of hepatic parenchyma, which are particularly susceptible to mechanical pressure of diaphragm. Another view that they have expressed states that there is a correspondence between the topography of the sulci and the course of the right and middle hepatic veins and their tributaries.

Cawich et al $(2016)^{14}$ have given an incidence $12 \%$ of fissures on the visceral surface of right lobe of liver in AfroCaribbean population. In the present study, these fissures were present in $53.8 \%$ livers to the right of porta-hepatis. This was much higher than $28 \%$ reported by Joshi et al. 5 They also observed the fissures to the right of fossa for gallbladder in $22 \%$ cases, which is in consonance with $25 \%$ found in our study. Further, they reported the fissures on the visceral surface of left lobe to be $18 \%$ which in our study was much lower, i.e. $8 \%$. Nayak $(2013)^{7}$ found a fissure on the left lobe only in $1.81 \%$ cases.

The incidence of fissures on caudate lobe was $44.23 \%$ in the present study, whereas that reported by Joshi et al ${ }^{5}$ was $30 \%$. These variations in the incidences reported may have more of clinical than morphological significance. The fissures on the quadrate lobe were seen in $55.77 \%$ of livers examined, whereas Joshi et al (2008) reported an incidence of $20 \%$.

CT findings by Auh et al (1984) ${ }^{9}$ regarding accessory fissures on the visceral surface of liver was $25 \%$, which increased to $70 \%$ with advancing age. Saritha et al ${ }^{11}$ found it in $30 \%$ cases. Cawich et al 14 have described the presence of accessory fissures in $12 \%$ livers. In general, the incidence of occurrence of accessory fissures in our study was $94.23 \%$. This may reflect that some of these smaller, superficial accessory fissures may not be properly delineated by CT or it could be because of population difference.

\section{CONCLUSION}

As very few studies on the incidence and pattern of accessory fissures of liver are available in the Central Indian population, these findings will be of great value in proper diagnosis and interpretation of USG or CT. These fissures may be confused with cyst or haematoma and may be the site of implantation of the secondaries. In an era of segmental resections, transplantation and laparoscopic surgeries the findings of the present study can be of great significance.

\section{REFERENCES}

1. Standrings S, Ellis H, Healy JC, et al. Gray's anatomy, abdominal viscera: liver: the anatomical basis of clinical practice. $14^{\text {th }}$ ed. Churchill livingstone 2008:1163-74.

2. Nagato AC, Silva MAS, Trajano ETL, et al. Quantitative and morphological analyses of different types of human liver. Jr of Morphological Sci 2011;28(4):275-9.

3. Singh R. Hypoplastic left lobe of liver with accessory caudate lobe. Case Reports in Medicine Article ID 604513, 2013;2013:3.

4. Auh YH, Lim JH, Wkim K, et al. Loculated fluid collections in hepatic fissures and recesses: CT appearance and potential pitfalls. Scientific- Exibit 1994;14(3):529-40.

5. Joshi SD, Joshi SS, Athavale SA. Some interesting observations on the surface features of the liver and their clinical implications. Singapore Med J 2009;50(7):715-9.

6. Sahni D, Jit I, Sodhi L, et al. Gross anatomy of caudate lobe of liver. JASI 1997;46(2):67-76.

7. Nayak SB. A study on the anomalies of liver in the South indian cadavers. Int J Morphol 2013;31(2):658-61.

8. Macchi V, Feltrin G, Parenti A, et al. Diaphragmatic sulci and portal fissures. J Anat 2003;202:303-8.

9. Auh YH, Rubenstein WA, Zirinsky K, et al. Accessory fissures of the liver: CT and sonographic appearance. AJR Am J Roentgenol 1984;143(3):565-72.

10. Patil S, Sethi M, Kakar M. Morphological study of human liver and its surgical importance. Int J Anat Res 2014;2(2):310-14.

11. Saritha $S$, Ramani, Nagajyothi, et al. Cadaveric study of morphological variations in the human liver and its clinical importance. International Journal Of Medical Science And Clinical Inventions 2015;2(6):1020-31.

12. Muktyaz H, Nema U, Suniti MR, et al. Anatomical study of accessory sulci of liver and its clinical significance in North Indian population. Int J Med Health Sci 2013;2(2):224-9.

13. Shetty $S$, kantha $L$, Jayanthi $V$, et al. A study of variant external features on cadaveric livers. Anatomica Karnataka 2011;5(3):12-6.

14. Cawich SO, Gardner MT, Shetty R, et al. Accessory inferior sulci of the liver in an afro- caribbean population. International Journal of Biomedical Science 2016; 12(2):58-64. 